\section{Response to Newman et al.}

To the Editor: We thank Dr Newman and colleagues ${ }^{1}$ for their careful reading of our article2 and for their additional recommendations.

We agree that creating a single set of guidelines covering the multitude of systemic and neurological manifestations of mitochondrial diseases is difficult, and that the recommendations will inherently not apply to every situation or every patient. However, the Delphi methodology we used allowed us to synthesize what were sometimes diverse views, and thereby to present a consensus based on the experience of a panel of mitochondrial disease physicians. This approach has been shown to be most valuable when there is limited objective evidence base, as for mitochondrial disorders.

In regard to the concern of whether a neuroophthalmologist is required for routine care of these patients, the writing group considered that such a specialist, if accessible by the patient, would be the ideal physician to monitor the ophthalmological manifestations of the disease. A neuro-ophthalmologist would also be the best person to determine the need for subspecialty ophthalmology referrals. It is important to note that this was only a recommendation, and not considered to be essential for each and every patient.

With regard to the monitoring of intraocular pressure, while it should be an integral part of every general eye exam, some members of the consortium have noted that this portion of the examination is not always performed in some pediatric patients. For this reason, an additional statement was included to recommend this testing be done when necessary.

We agree that lubrication is required to prevent the keratopathy that occurs with corneal exposure in some mitochondrial patients, and not only due to the inappropriate spread of tears. We also agree that it would be wise for affected and unaffected Leber hereditary optic neuropathy (LHON) mitochondrial DNA mutation carriers to avoid cigarette smoking and excessive alcohol use, although the evidence that well-established LHON deteriorates with smoking or excess alcohol is limited. The seventh recommendation was included to alert the clinician to the presence of LHON plus phenotypes, with some authors feeling that a periodic neurologic exam may detect preclinical symptoms, and a follow-up electrocardiogram may detect a preexcitation syndrome not apparent on the first examination.

It is important to stress that our recommendations were not a universal view held by all of the authors but a consensus statement reflecting the majority. This approach has both strengths and weaknesses. Inevitably this means that the outcome of the review was dependent on the authors who engaged the process. To address this, we made an open invitation to interested clinician-members of the Mitochondrial Medicine Society to participate. However, most importantly, the consensus describes a "current state of play," which definitely should be revised as additional objective information comes to hand.

We therefore welcome their additional comments, and look forward to incorporating these suggestions in any future revised version of the criteria.

\section{DISCLOSURE}

The authors declare no conflict of interest.

Sumit Parikh, $M D^{1}$, Amy Goldstein, $M D^{2}$, Amel Karaa, $M D^{3}$, Mary Kay Koenig, $M D^{4}$, Irina Anselm, $M D^{5}$,

Catherine Brunel-Guitton, MD, FRCPC 6

John Christodoulou, MBBS, $P h D^{7}$, Bruce H. Cohen, $M D^{8}$,

David Dimmock, $M D^{9}$, Gregory M. Enns, $M B, C h B^{10}$,

Marni J. Falk, $M D^{11}$, Annette Feigenbaum, $M D^{12,13}$,

Richard E. Frye, $M D, P h D^{14}$, Jaya Ganesh, $M D^{15}$,

David Griesemer, $M D^{16}$, Richard Haas, $M B, B C h i r, M R C P^{17,18}$ , Rita Horvath, $M D, P h D^{19}$, Mark Korson, $M D^{20}$,

Michael C. Kruer, $M D^{21}$, Michelangelo Mancuso, $M D, P h D^{22}$, Shana McCormack, $M D^{23}$, Marie Josee Raboisson, $M D^{24}$,

Tyler Reimschisel, $M D, M H P E^{25}$,

Ramona Salvarinova, $M D, F R C P C^{26}$,

Russell P. Saneto, DO, PhD $D^{27}$, Fernando Scaglia, $M D^{28}$,

John Shoffner, $M D^{29}$, Peter W. Stacpoole PhD, $M D^{30}$, Carolyn M. Sue, MBBS, $P h D^{31}$, Mark Tarnopolsky, $M D, P h D^{32}$, Clara Van Karnebeek, $M D, P h D^{33,34}$,

Lynne A. Wolfe, MS, CRNP ${ }^{35}$,

Zarazuela Zolkipli Cunningham, $M B C h B, M R C P^{36}$,

Shamima Rahman, FRCP, $P h D^{37}$ and

Patrick F. Chinnery, FRCP, FMedSci ${ }^{38}$

${ }^{1}$ Center for Child Neurology, Cleveland Clinic Children's Hospital, Cleveland, Ohio, USA; ${ }^{2}$ Division of Child Neurology, Children's Hospital of Pittsburgh, Pittsburgh, Pennsylvania, USA; ${ }^{3}$ Division of Genetics, Massachusetts General Hospital, Boston, Massachusetts, USA; ${ }^{4}$ Division of Child and Adolescent Neurology, University of Texas Medical School at Houston, Houston, Texas, USA; ${ }^{5}$ Department of Neurology, Boston Children's Hospital, Boston, Massachusetts, USA; ${ }^{6}$ Department of Pediatrics, University of Montreal, Montreal, Quebec, Canada; ${ }^{7}$ Neurodevelopmental Genomics Research Group, Murdoch Childrens Research Institute, and Department of Paediatrics, Melbourne Medical School, University of Melbourne, Melbourne, Victoria, Australia; ${ }^{8}$ Neurodevelopmental Science Center, Children's Hospital Medical Center of Akron, Akron, Ohio, USA; ${ }^{9}$ Rady Children's Institute for Genomic Medicine, San Diego, California, USA; ${ }^{10}$ Division of Medical Genetics, Department of Pediatrics, Stanford University Lucile Packard Children's Hospital, Palo Alto, California, USA; ${ }^{11}$ Division of Human Genetics, Department of Pediatrics, The Children's Hospital of Philadelphia and University of Pennsylvania Perelman School of Medicine, Philadelphia,

Pennsylvania, USA; ${ }^{12}$ Division of Clinical and Metabolic Genetics, The Hospital for Sick Children and University of Toronto, Toronto, Ontario, Canada; ${ }^{13}$ Department of Pediatrics, University of California San Diego and Rady Childrens Hospital, San Diego, California, USA; ${ }^{14}$ Department of Pediatrics, University of Arkansas Medical Sciences, Little Rock, Arkansas, USA; ${ }^{15}$ Division of Genetics, Department of Pediatrics, Cooper Medical School at Rowan University, Camden, New Jersey, USA; ${ }^{16}$ Division of Neurology, Levine Children's Hospital, Charlotte, North Carolina, USA; ${ }^{17}$ Departments of Neurosciences and Pediatrics, University of California San Diego, La Jolla, California, USA; ${ }^{18}$ Department of Neurosciences, Rady Children's Hospital, San Diego, California, USA; ${ }^{19}$ Institute of Genetic Medicine, Newcastle University, Newcastle upon Tyne, UK; ${ }^{20}$ Genetic Metabolic Center for Education, Salem, Massachusetts, USA; ${ }^{21}$ Department 
of Pediatric Neurology, University of Arizona College of Medicine, Phoenix, Arizona, USA; ${ }^{22}$ Department of Experimental and Clinical Medicine, Neurological Clinic, University of Pisa, Pisa, Italy; ${ }^{23}$ Division of Endocrinology and Diabetes, Children's Hospital of Philadelphia, Philadelphia, Pennsylvania, USA; ${ }^{24}$ Department of Cardiology, CHU Sainte-Justine, Montreal, Quebec, Canada; ${ }^{25}$ Department of Pediatrics, Vanderbilt University Medical Center, Nashville, Tennessee, USA; ${ }^{26}$ Division of Biochemical Diseases, BC Children's Hospital, Vancouver, British Columbia, Canada; ${ }^{27}$ Department of Neurology, Seattle Children's Hospital/University of Washington, Seattle, Washington, USA; ${ }^{28}$ Department of Molecular and Human Genetics, Baylor College of Medicine and Texas Children's Hospital, Houston, Texas, USA; ${ }^{29}$ Neurology, Biochemical \& Molecular Genetics, Atlanta, Georgia, USA; ${ }^{30}$ Department of Medicine, University of Florida College of Medicine, Gainesville, Florida, USA; ${ }^{31}$ Department of Neurology and Kolling Institute, Royal North Shore Hospital, St Leonards, New South Wales, Australia; ${ }^{32}$ Division of Neurology, McMaster University, Hamilton, Ontario, Canada; ${ }^{33}$ Department of Pediatrics, Academic Medical Centre, University of Amsterdam, Amsterdam, The Netherlands; ${ }^{34}$ Department of Pediatrics, Centre for Molecular Medicine, University of British Columbia, Vancouver, British Columbia, Canada; ${ }^{35}$ Undiagnosed Diseases Network, National Institutes of Health, Bethesda, Maryland, USA; ${ }^{36}$ Division of Neurology, The Children's Hospital of Philadelphia, Philadelphia, Pennsylvania, USA ${ }^{37}$ Mitochondrial Research Group, UCL
Great Ormond Street Institute of Child Health, London, UK; ${ }^{38}$ Department of Clinical Neurosciences \& MRC Mitochondrial Biology Unit, University of Cambridge,

Cambridge, UK. Correspondence: Sumit Parikh (parikhs@ccf.org)

\section{REFERENCES}

1. Newman NJ, Yu-Wai-Man P, Sadun AA,. Karanjia R, Carelli V. Management of ophthalmologic manifestations of mitochondrial diseases. Genet Med; E-pub ahead of print 26 October, 2017.

2. Parikh S, Goldstein A, Karaa A, et al. Patient care standards for primary mitochondrial disease: a consensus statement from the Mitochondrial Medicine Society [online only]. Genet Med 2017. doi:10.1038/ gim.2017.107.

Advance online publication 26 October 2017. doi:10.1038/gim.2017.164 